\title{
Joints and Fascia Progression GvHD
}

National Cancer Institute

\section{Source}

National Cancer Institute. Joints and Fascia Progression GVHD. NCI Thesaurus. Code C126734.

A NIH joint and fascia score that has increased by 1 or more points or a decrease in PROM score by 1 point for any site. 